\title{
Acceptability, Compliance, and Safety of Non-small Cell Lung Cancer Cachectic Participants Continuing Compassionate Access in the ACCeRT Clinical Study
}

\author{
Elaine S Rogers, Ph.D. ${ }^{1,2}$, Rita Sasidharan, MBBS. ${ }^{2}$, Graeme M Sequeira, M.Sc. ${ }^{3}$, \\ Matthew R Wood, M.Sc. ${ }^{4}$, Stephen P Bird, Ph.D. ${ }^{5}$, Justin W L Keogh, Ph.D. ${ }^{6}$, \\ Bruce Arroll, Ph.D. ${ }^{1}$, Joanna Stewart, M.Sc. ${ }^{7}$, Roderick D MacLeod, Ph.D. ${ }^{8}$
}

'Department of General Practice and Primary Health Care, University of Auckland, Auckland, 1142 New Zealand. ${ }^{2}$ Department of Medical Oncology, Auckland City Hospital, Auckland, 1023 New Zealand.

${ }^{3}$ School of Sport, Manukau Institute of Technology, Auckland, 2241 New Zealand.

${ }^{4}$ Sports Performance Research Institute New Zealand (SPRINZ), Auckland University of Technology, Auckland, 1010 New Zealand. ${ }^{5}$ Department of Medical and Exercise Science, University of Wollongong, New South Wales, 2522 Australia.

${ }^{6}$ Faculty of Health Sciences and Medicine, Bond University, Gold Coast, 4226 Australia.

${ }^{7}$ Department of Epidemiology and Biostatistics, University of Auckland, Auckland, 1142 New Zealand.

${ }^{8}$ Northern Clinical School, University of Sydney, Sydney, New South Wales, 2065 Australia.

Received 27 April 2021 • Revised 11 July 2021 • Accepted 12 July 2021 • Published online 5 October 2021

\begin{abstract}
:
Objective: Cancer cachexia is defined as: a 'multifactorial syndrome', and it has been suggested that a multitargeted approach is required in its management. High prevalence is seen within non-small cell lung cancer, and patients may continue to experience cachexia post end of anti-cancer treatment, and in the late/end stage.

Material and Methods: Participants who had completed week 20/End of Trial visit in the main Auckland's Cancer Cachexia evaluating Resistance Training (ACCeRT) study were invited to continue with treatment under compassionate use. Participants could continue with $2.09 \mathrm{~g}$ of eicosapentaenoic acid (EPA), $300 \mathrm{mg}$ COX-2 inhibitor (celecoxib), once daily; plus two sessions per week of progressive resistance training (PRT), and $20 \mathrm{~g}$ oral essential amino acids (EAA); high in leucine, in a split dose over three days post each session. Data was collected on the acceptability, compliance and adherence to medication/PRT sessions. Secondary endpoints included: change in body weight and fat free mass,

Contact: Elaine S Rogers, Ph.D.

Department of General Practice and Primary Health Care, University of Auckland, Auckland, 1142 New Zealand.

Department of Medical Oncology, Auckland City Hospital, Auckland, 1023 New Zealand.

E-mail: es.rogers@auckland.ac.nz

(c) 2021 JHSMR. Hosting by Prince of Songkla University. All rights reserved.

This is an open access article under the CC BY-NC-ND license

(http://www.jhsmr.org/index.php/jhsmr/about/editorialPolicies\#openAccessPolicy).

J Health Sci Med Res 2022;40(3):335-347 doi: $10.31584 /$ jhsmr.2021842 www.jhsmr.org
\end{abstract}


handgrip and leg strength, the Functional Assessment of Anorexia/Cachexia Therapy, Multidimensional Fatigue Symptom Inventory-Short Form, World Health Organization Quality of Life — BREF, Glasgow prognostic score, and pro-inflammatory cytokines.

Results: All six participants, who completed the main ACCeRT study, opted to continue with compassionate use. Acceptability remained high, with overall compliance to last study/PRT visit of $81.0 \%$ for EPA, $98.8 \%$ for celecoxib, $78.9 \%$ for PRT and $77.2 \%$ for EAA. Participants continued to lose body weight and Fat-Free Mass, along with reduced albumin and increased C-Reactive protein levels. Mean time on compassionate study treatment was 78 days, and with a mean overall survival of 257 days $(140+117)$.

Conclusion: Non-small cell lung cancer (NSCLC) cachectic patients are willing to be enrolled onto a multi-targeted treatment regimen, and may benefit from cachexia symptom management even during their late/refractory stage.

Keywords: Refractory cancer cachexia, Resistance Training, NSCLC cachectic patients, multi-targeted treatment

\section{Introduction}

Cachexia still remains a challenge within the oncology population. A recent, cross-sectional study investigated the prevalence of cancer cachexia in 386 , of 426 eligible patients attending selected hospitals within Norway. ${ }^{1}$ Prevalence of $21.0 \%$ was seen amongst outpatients; increasing to $51.0 \%$ within the inpatient population. High levels were again reported within the lung cancer population, with $36.0 \%$ amongst outpatients and increasing to $83.0 \%$ of inpatients. Patients were asked if they required either decreased or increased attention to their weight. Fifteen percent of outpatients and $20.0 \%$ of inpatients requested: "more or a lot more focus", on this condition. ${ }^{1}$

It has long been established that cachectic cancer patients have been associated and/or documented to have lower response rates to traditional chemotherapy as well as shorter median survival, ${ }^{2}$ which has been attributed to a number of factors. Firstly, because chemotherapy dosage is based on body surface area, thinner patients receive a lower dose of chemotherapy. Secondly, treatment breaks, due to chemotherapy toxicities, are higher in this population of patients. ${ }^{3}$ Thirdly, previous chemotherapy treatments and concurrent multi-modality treatments e.g., surgery and radiotherapy will also affect nutritional status, which further confound the condition. A retrospective review suggested that metastatic melanoma patients treated with chemotherapy, immunotherapy and targeted therapy with a body mass index (BMI) of either overweight or obese, as classified by the World Health Organisation (WHO), had improved progression-free survival and overall survival when compared with patients of normal weight. ${ }^{4}$ Improved survival within early obese cancer patients has been termed as the: 'obesity paradox', and has been shown in a number of different cancers. ${ }^{6}$ Failure to respond to pembrolizumab has been recently identified, due to elevated protein catabolism and clearance seen within cancer cachectic patients. ${ }^{7,8}$ With the increase in the use of immune checkpoint inhibitors that have revolutionized the current treatments in oncology, this highlights the need to diagnose and support cachectic patients throughout their anti-cancer treatments and beyond.

Cancer cachexia has recently been defined as multifactorial; including, elements of decreased total body weight, adjoined with skeletal muscle loss contributing to impaired function, respiratory complications and fatigue, decreased food intake, metabolic changes, increased inflammation and catabolism; affecting the patients' overall quality of life. , $^{10}$ 
Multi-targeted/multi-modal studies have been designed to address these factors, and recently the results of the Pre-Multimodal Exercise/Nutrition/Anti-inflammatory Treatment for Cachexia (Pre-MENAC) study have been published. This study investigated the use of standard cancer care versus a multi-modal regimen, comprising of a single baseline session of nutritional counselling (approximately 30 minutes), eicosapentaenoic acid (EPA) via two $220 \mathrm{~mL}$ cartons of oral nutritional supplement (Prosure @ Abbott) equaling a net intake of $2 \mathrm{~g} /$ day, $300 \mathrm{mg}$ celecoxib OD, twice weekly home-based aerobic sessions (approximately 30 minutes) and thrice weekly resistance training of six individualized exercises (approximately 20 minutes). The study period of six weeks ran concurrently, with cycles I and II of chemotherapy in non-small cell lung cancer (NSCLC), and pancreatic cancer patients with less than $20.0 \%$ weight loss over the preceding six months. ${ }^{11}$ This study recruited pre-cachexia/cachexia patients, with the results demonstrating no significant difference between groups on muscle mass, as assessed by computerized tomography (CT) derived measures, or physical activity assessed by 6MWT and ActivPAL. Compliance rates of $60.0 \%$ for exercise, $48.0 \%$ for nutritional supplement and $76.0 \%$ for celecoxib were observed. ${ }^{11}$

Anamorelin is the only registered drug for the treatment of cancer cachexia recently approved by Japan in December 2020. ${ }^{12}$ The intervention(s) being tested within the ACCeRT study are an alternative treatment regimen.

The study was designed to address cachectic factors within a multi-targeted regimen, within endstage/refractory cachectic NSCLC patients. ${ }^{13}$ The study combination was chosen to increase muscle anabolism, using progressive resistance training (PRT) and essential amino acids (EAA) high in leucine, post exercise as well as to target and decrease the proinflammatory cytokines by using a cyclooxygenase-2 inhibitor (celecoxib) and EPA. Results from the main study showed high acceptability, and compliance rates of $86.8 \%$ for EPA, $100.0 \%$ for celecoxib, 94.4\% for PRT/exercise, and $76.5 \%$ for EAA within the treatment group at week 20. Trends in efficacy, in terms of improvement and/or stability in cachexia markers, were seen within magnetic resonance imaging (MRI) muscle volume, albumin, and C-reactive protein (CRP) levels within both arms. ${ }^{14}$ Participants who had completed the ACCeRT main study could continue with medication and/or exercise sessions post end of the main study under compassionate use (CU). Data on the ongoing acceptability, compliance and safety of this multi-targeted regimen in refractory cachectic NSCLC patients will be used to calculate the power and number of participants required for a future phase II study.

\section{Material and Methods}

Northern $Y$ Ethics Committee, Hamilton, New Zealand (NTY/11/06/064) approved the published study protocol. ${ }^{13}$ The study was registered with the Australian and New Zealand Clinical Trials Registry, and complied with the International Ethical Guidelines for Biomedical Research Involving Human Subjects, Good Clinical Practice Guidelines, and the Declaration of Helsinki. Participants consisted of those whom had completed week 20/End of Trial (EOT) visit in the main ACCeRT study, had maintained an Eastern Cooperative Oncology Group Performance Status (ECOGPS) $\leq 2$, and for which the investigator considered suitable to continue to receive additional treatments and exercise sessions. Participants were permitted to withdraw at any time, or at the discretion of the investigator; due to further progression of their disease.

\section{Procedures}

All participants could choose to continue to orally receive $2.09 \mathrm{~g}$ of EPA, plus $300 \mathrm{mg}$ of celecoxib once daily. Arm A participants could choose to commence with the addition of two PRT sessions per week (Tuesdays and Fridays), followed by $20 \mathrm{~g}$ EAA high in leucine in split 
doses over the following three days. Arm B participants could choose to continue with PRT/EAA. Dose reductions or interruptions of all study medications, and exercise sessions were permitted. The intention of compassionate PRT differed depending on study randomisation to either Arm A or Arm B within the main study. Those participants that had completed the 20-week PRT programme (Arm B) had the option to continue with resistance training. The intention for this group of participants was to maintain the same training intensity and volume achieved at week 20/ EOT of the PRT programme. Those participants that were not allocated to the PRT exercise group (Arm A) had the option of starting the PRT programme, using the same exercise programme designed from the main study. All participants were medically assessed every four weeks.

\section{Outcomes}

Data was collected on the acceptability of a multitargeted regimen of supportive care in cachectic NSCLC participants continuing with compassionate use. Acceptability was assessed by the analysis of a patient rated Likert scored questionnaire, asking 10 questions on the acceptability of the above multi-targeted approach. Likert scores had a range of five, five being: 'strongly agree', and one being: 'strongly disagree', with higher scores representing higher acceptability of the study medication and/or exercise programme. Secondary endpoints were continued over from the main study, and included the change from baseline in body composition (fat-free mass (FFM), total body weight, and fat mass), as measured by Bioelectrical Impedance Analysis (BIA) (Tanita), muscle strength (hand grip dynamometry and isometric knee extension, measured by the use of a customised rig attached to a load cell). Symptom burden was measured with the anorexia-cachexia scale and physical well-being scale from the Functional Assessment of Anorexia/Cancer Therapy (version 4).
Fatigue was measured by The Multidimensional Fatigue Symptom Inventory-Short Form, and overall quality of life by WHOQOL-BREF. Proinflammatory cytokine analysis (IL-1 $\beta, I L-6$, and $T N F-\alpha)$ was measured by Luminex MAGPIX ${ }^{\circledR}$. Albumin and CRP levels were incorporated into the Glasgow Prognostic Score. Compliance results were analysed as percentage of the total study medication, and percentage of attendance of the total study PRT sessions. All the above data were collected every four weeks, and study participants were followed up for overall survival

\section{Results}

Sixty-nine patients were screened, resulting in 20 patients being enrolled onto the main study (Figure 1 Consolidated Standards of Reporting Trials profile). All six participants, completing week 20/EOT visit from the main study continued with study medication, and either continued/ began PRT sessions and EAA under CU (two allocated to $\operatorname{Arm~A,~and~four~allocated~to~Arm~B).~Baseline~characteristic~}$ data is shown in Table 1.

The acceptability questionnaire was completed at each monthly $\mathrm{CU}$ visit, as shown in Table 2. Results showed high mean acceptability scores of 4 to 5 for EPA, celecoxib, PRT and EAA, and commitment to continue with the regimen from $\mathrm{CU} 1$ through to $\mathrm{CU} 5$ visit.

Compliance data is shown in Table 3 (deemed as $>50.0 \%$ for each participant) and was $80.0 \%(n=4 / 5)$ for EPA, $100.0 \%(n=5 / 5)$ for celecoxib, $83.3 \%(n=5 / 6)$ for the PRT component, and $75.0 \%(n=3 / 4)$ for EAA.

Overall compliance was $81.0 \%$ for EPA, $98.8 \%$ for celecoxib, $78.9 \%$ for PRT, and $77.2 \%$ for EAA; as shown in Table 4. These results conclude that on average, the administration of EPA, celecoxib, PRT and EAA at this dose and frequency was acceptable within this population; with three of the six participants continuing with all four elements of the multi-targeted regimen. 


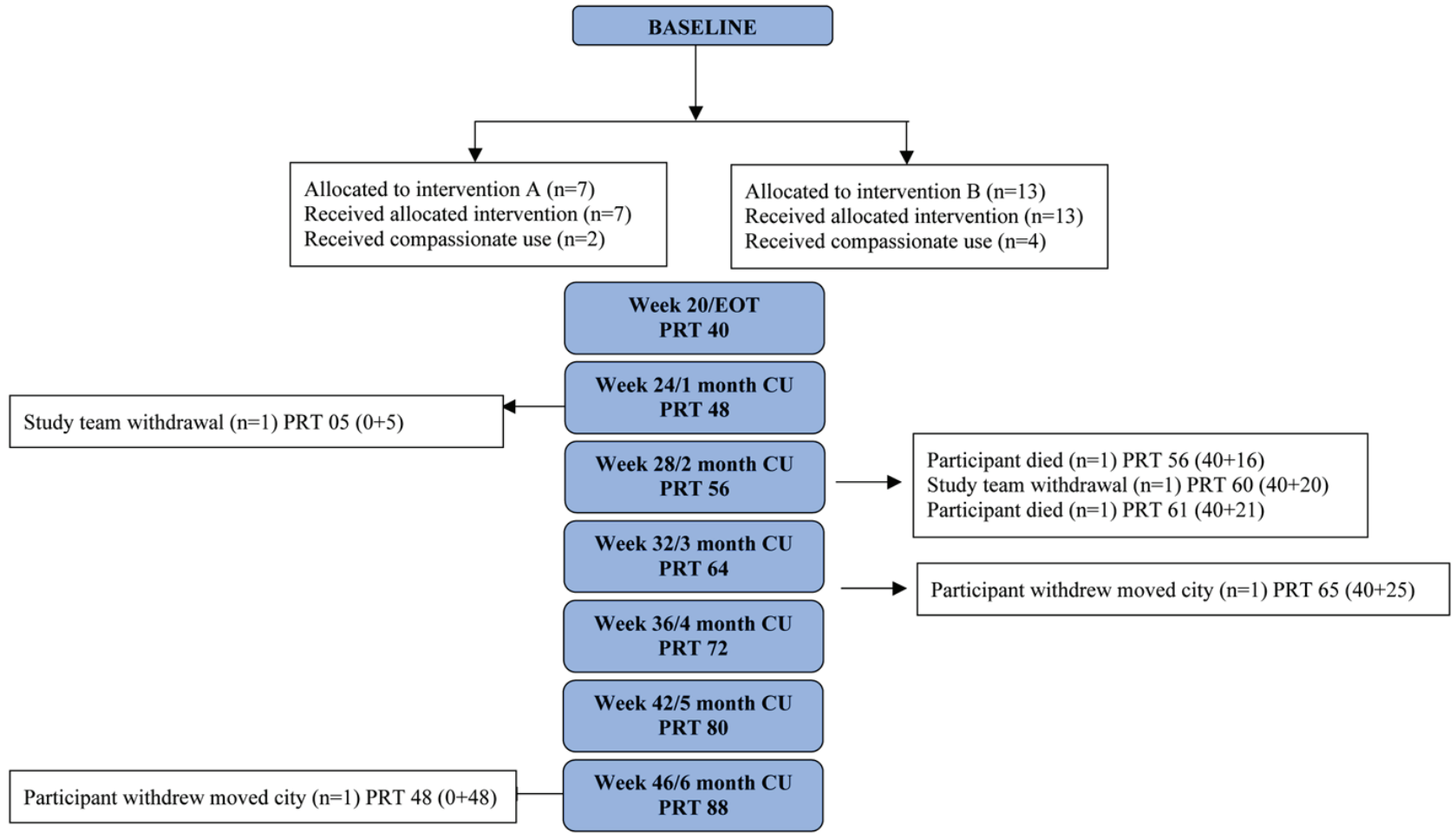

$\mathrm{PRT}=$ progressive resistance training, $\mathrm{CU}=$ compassionate use, $\mathrm{EOT}=\mathrm{end}$ of trial

Figure 1 Consolidated Standards of Reporting Trials

Table 1 Auckland's Cancer Cachexia evaluating Resistance Training compassionate use Baseline data/characteristics

\begin{tabular}{|c|c|c|}
\hline & \multicolumn{2}{|c|}{$\begin{array}{c}\text { Total } \\
\text { Mean (range) } \\
n=6\end{array}$} \\
\hline & At ACCeRT entry & At ACCeRT CU entry \\
\hline Age (years) & 63.7 (42 to 71$)$ & \\
\hline \multicolumn{3}{|l|}{ Race } \\
\hline European & 4 & \\
\hline Māori & 2 & \\
\hline \multicolumn{3}{|l|}{ Gender } \\
\hline Male & 4 & \\
\hline Female & 2 & \\
\hline \multicolumn{3}{|l|}{ Weight loss (\%) } \\
\hline All & $-7.9(-5.0 \text { to }-9.8)^{\mathrm{a}}$ & $-3.7(-10.2 \text { to }+4.4)^{\mathrm{b}}$ \\
\hline 0.0 to $5.0 \%$ & 0 & 2 \\
\hline 5.0 to $10.0 \%$ & 5 & 3 \\
\hline+0.0 to $+5.0 \%$ & 0 & 1 \\
\hline Low BMI & $1^{a}$ & 0 \\
\hline
\end{tabular}


Table 1 (continued)

\begin{tabular}{|c|c|c|}
\hline & \multicolumn{2}{|c|}{$\begin{array}{c}\text { Total } \\
\text { Mean (range) } \\
n=6\end{array}$} \\
\hline & At ACCeRT entry & At ACCeRT CU entry \\
\hline \multicolumn{3}{|l|}{ Body weight (kg) } \\
\hline All & $72.8(45.1 \text { to } 89.1)^{\mathrm{C}}$ & $69.7(47.1 \text { to } 87.7)^{d}$ \\
\hline Male & $78.3(70.7 \text { to } 89.1)^{\mathrm{C}}$ & $74.1(66.7 \text { to } 87.7)^{d}$ \\
\hline Female & $61.9(45.1,78.6)^{\mathrm{C}}$ & $60.9(47.1,74.6)^{d}$ \\
\hline Time since diagnosis (days) & $429(125 \text { to } 920)^{e}$ & \\
\hline \multicolumn{3}{|l|}{ Diagnosis NSCLC } \\
\hline Adenocarcinoma & 4 & \\
\hline Squamous & 2 & \\
\hline Albumin (g/) & $37.5(25 \text { to } 44)^{\mathrm{C}}$ & $34.8(27 \text { to } 40)^{d}$ \\
\hline $\mathrm{CRP}(\mathrm{mg} /)$ & $37.8(5 \text { to } 62)^{c}$ & $86.3(2 \text { to } 211)^{d}$ \\
\hline GPS & $0.8(0 \text { to } 2)^{\circ}$ & $1.2(0 \text { to } 2)^{d}$ \\
\hline
\end{tabular}

${ }^{a}$ Weight loss defined as percentage weight loss at main ACCeRT study entry. *one participant study entry with low BMI.

beight loss/gain defined as weight change from Random visit to week 20/EOT visit of main ACCeRT study.

'Data at study Random visit.

${ }^{\mathrm{d} D a t a}$ at week 20/EOT visit/start of CU.

${ }^{\mathrm{e}}$ Time since diagnosis defined as histology date to date of main ACCeRT study entry.

$\mathrm{CU}=$ compassionate use, $\mathrm{BMI}=$ body mass index, NSCLC=non-small cell lung cancer, $\mathrm{CRP}=\mathrm{C}$-reactive protein, GPS=glasgow prognostic score, EOT=end of trial, ACCeRT=Auckland's Cancer Cachexia evaluating Resistance Training

Table 2 Acceptability questionnaire results

\begin{tabular}{llllll}
\hline & CU 1 & CU 2 & CU 3 & CU 4 CU 5 \\
\hline EPA acceptable (5) & $5^{\mathrm{a}}$ & $5^{\mathrm{a}}$ & & & \\
Celebrex acceptable (5) & $5^{\mathrm{b}}$ & $5^{\mathrm{b}}$ & $4.5^{\mathrm{a}}$ & $5^{\mathrm{c}}$ & $5^{\mathrm{c}}$ \\
PRT acceptable (5) & $5^{\mathrm{b}}$ & $5^{\mathrm{b}}$ & $4.5^{\mathrm{a}}$ & $5^{\mathrm{c}}$ & $5^{\mathrm{c}}$ \\
EAA acceptable (5) & $5^{\mathrm{a}}$ & $5^{\mathrm{a}}$ & $4^{\mathrm{c}}$ & & \\
Continue with exercise and medication (5) & $5^{\mathrm{b}}$ & $5^{\mathrm{b}}$ & $4.5^{\mathrm{a}}$ & $5^{\mathrm{c}}$ & $5^{\mathrm{c}}$ \\
Total number of participants in the study & 5 & 5 & 2 & 1 & 1 \\
Total number of participants in the study with data & 4 & 3 & 2 & 1 & 1 \\
\hline
\end{tabular}

${ }^{a} \mathrm{n}=2$ participants taking either the medication/exercise.

${ }^{b} \mathrm{n}=3$ participants taking either the medication/exercise.

${ }^{c} \mathrm{n}=1$ participant taking either the medication/exercise.

Highest score available for each question within parenthesis.

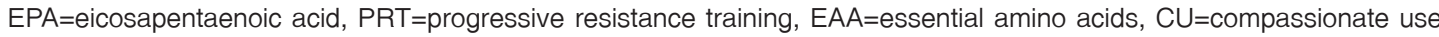

One Arm A and one Arm B particpant did not complete the acceptability questionaire during compassionate use (CU). 
Table 3 Compliance table for individual participants completing compassionate use

\begin{tabular}{|c|c|c|c|c|c|}
\hline & \multicolumn{5}{|c|}{ Percentage taken of the total study dose/attendance at sessions } \\
\hline & EPA & Celecoxib & PRT/Days & PRT & EAA \\
\hline \multirow[t]{2}{*}{ Arm A } & 100 & 0 & 5 sessions & 100 & 21 \\
\hline & $25 / 25$ days & 0 & 25 days & $5 / 5$ & $21 / 100 \mathrm{~g}$ \\
\hline \multirow[t]{2}{*}{ Arm A } & 4.8 & 93.9 & 48 sessions & 79.2 & 0 \\
\hline & 8 days* & 155 days* & 165 days & $38 / 48$ & 0 \\
\hline \multirow[t]{2}{*}{ Arm B } & 100 & 100 & 16 sessions & 81.3 & 93 \\
\hline & $53 / 53$ days & $53 / 53$ days & 53 days & $13 / 16$ & $186 / 200 \mathrm{~g}^{*}$ \\
\hline \multirow[t]{2}{*}{ Arm B } & 100 & 100 & 21 sessions & 85.7 & 100 \\
\hline & $71 / 71$ days & $71 / 71$ days & 71 days & $18 / 21$ & $420 / 420 \mathrm{~g}$ \\
\hline \multirow[t]{2}{*}{ Arm B } & 0 & 100 & 25 sessions & 32 & 0 \\
\hline & 0 & $87 / 87$ days & 87 days & $8 / 25$ & 0 \\
\hline \multirow[t]{2}{*}{ Arm B } & 100 & 100 & 20 sessions & 95 & 94.7 \\
\hline & $67 / 67$ days & $67 / 67$ days & 67 days & $19 / 20$ & $360 / 380 \mathrm{~g}$ \\
\hline
\end{tabular}

*participant decision to stop

CU 1 equals 28 doses of EPA and celecoxib, 8 PRT sessions, and $160 \mathrm{~g}$ of EAA.

CU 2 equals 56 doses of EPA and celecoxib, 16 PRT sessions, and $320 \mathrm{~g}$ of EAA.

CU 3 equals 84 doses of EPA and celecoxib, 24 PRT sessions, and $480 \mathrm{~g}$ of EAA.

CU 4 equals 112 doses of EPA and celecoxib, 32 PRT sessions, and $640 \mathrm{~g}$ of EAA.

CU 5 equals 140 doses of EPA and celecoxib, 40 PRT sessions, and $800 \mathrm{~g}$ of EAA.

$\mathrm{EPA}=$ eicosapentaenoic acid, $\mathrm{PRT}=$ progressive resistance training, $\mathrm{EAA}=$ essential amino acids, $\mathrm{CU}=\mathrm{compassionate}$ use

Table 4 Percentage compliance table for participants completing compassionate use

\begin{tabular}{lllll}
\hline & EPA & Celecoxib & PRT & EAA \\
\hline Arm A & 100 & & 100 & 21 \\
Arm A & 4.8 & 93.9 & 79.2 & \\
Arm B & 100 & 100 & 81.3 & 93 \\
Arm B & 100 & 100 & 85.7 & 100 \\
Arm B & & 100 & 32 & \\
Arm B & 100 & 100 & 95 & 94.7 \\
Mean & $\mathbf{8 1 . 0 ^ { \mathbf { a } }}$ & $\mathbf{9 8 . 8 ^ { \mathrm { b } }}$ & $\mathbf{7 8 . 9}$ & $\mathbf{7 7 . 2 ^ { \mathrm { c } }}$ \\
\hline
\end{tabular}

${ }^{a} \mathrm{n}=5$ participants taking EPA. One previous Arm B participant declined EPA.

${ }^{b} \mathrm{n}=5$ participants taking celecoxib. One previous Arm A participant did not take celecoxib, due to remaining on diclofenac $100 \mathrm{mg}$ sustained release.

${ }^{c} n=4$ participants taking EAA. One previous Arm A and one previous Arm $B$ declined EAA.

$\mathrm{EPA}=$ eicosapentaenoic acid, PRT=progressive resistance training, $E A A=e s s e n t i a l$ amino acids, $C U=$ compassionate use

\section{Secondary endpoints}

Individual participant mean weight, FFM, albumin and CRP level, per each study visit, is shown in Table 5. Three participants continued to lose total body weight, and all four participants continued to lose FFM throughout $\mathrm{CU}$ visits. At CU 1 (main study week 20 + week $4 \mathrm{CU}=24$ weeks) there was a mean percentage weight change of $-5.6 \%$ (range -11.5 to $+5.1 \%, n=3$ ), with a mean FFM change of $-3.2 \%$ (range -7.0 to $+1.8 \%, n=3$ ). At CU 2 (28 weeks) there was a mean percentage weight change of $-6.5 \%$ (range -11.0 to $+2.2 \%, n=3$ ), with a mean FFM change of $-3.4 \%$ (range -4.6 to $-1.5 \%, n=3$ ). At CU 3 (32 weeks) there was a mean percentage weight change of $-6.7 \%(-12.0 \%,-1.3 \%, n=2)$, with a mean FFM change of $-4.7 \%(-8.1 \%,-1.3 \%, n=2)$. For the remaining participant results at CU 4 (36 weeks) there was a percentage weight change of $-13.0 \%$, and a FFM change of $-7.7 \%$. At CU 5 (40 weeks) there was a 
percentage weight change of $-12.9 \%$, and a FFM change of $-4.6 \%$. This indicates relatively low FFM loss of $-3.2 \%$ to $-4.6 \%$ over the 20 -week main study, plus a further 4 to 20 weeks during compassionate use.

Table 5 Individual participants secondary outcome results

\begin{tabular}{|c|c|c|c|c|c|c|c|c|}
\hline Arm A & & Randomisation & Week 20 & CU 1 & CU 2 & CU 3 & CU 4 & CU 5 \\
\hline \multirow[t]{3}{*}{ Weight (kg) } & & 70.7 & 68.2 & 62.6 & 62.9 & 62.2 & 61.5 & 61.6 \\
\hline & Difference & & -2.5 & -8.1 & -7.8 & -8.5 & -9.2 & -9.1 \\
\hline & $\%$ difference & & $-3.5 \%$ & $-11.5 \%$ & $-11.0 \%$ & $-12.0 \%$ & $-13.0 \%$ & $-12.9 \%$ \\
\hline \multirow[t]{3}{*}{ FFM (kg) } & & 54.6 & 53.0 & 50.8 & 52.3 & 50.2 & 50.4 & 52.1 \\
\hline & Difference & & -1.6 & -3.8 & -2.3 & -4.4 & -4.2 & -2.5 \\
\hline & $\%$ difference & & $-3.0 \%$ & $-7.0 \%$ & $-4.2 \%$ & $-8.1 \%$ & $-7.7 \%$ & $-4.6 \%$ \\
\hline \multirow[t]{3}{*}{ Albumin ( $g /$ ) } & & 35 & 35 & 28 & 30 & 34 & 35 & 33 \\
\hline & Difference & & 0 & -7 & -5 & -1 & 0 & -2 \\
\hline & $\%$ difference & & $0.0 \%$ & $-20.0 \%$ & $-14.3 \%$ & $-2.9 \%$ & $0.0 \%$ & $-5.7 \%$ \\
\hline \multirow[t]{3}{*}{ CRP (mg/) } & & 9 & 12 & 32 & 30 & 13 & 32 & 27 \\
\hline & Difference & & +3 & +23 & +21 & +4 & +23 & +18 \\
\hline & $\%$ difference & & $+33.3 \%$ & $+255.6 \%$ & $+233.3 \%$ & $+44.4 \%$ & $+255.6 \%$ & $+200.0 \%$ \\
\hline Arm B & & Randomisation & Week 20 & CU 1 & $\mathrm{CU} 2$ & $\mathrm{CU} 3$ & CU 4 & CU 5 \\
\hline \multirow[t]{3}{*}{ Weight (kg) } & & 78.9 & 73.9 & 70.7 & 70.4 & & & \\
\hline & Difference & & -5.0 & -8.2 & -8.5 & & & \\
\hline & $\%$ difference & & $-6.3 \%$ & $-10.4 \%$ & $-10.8 \%$ & & & \\
\hline \multirow[t]{3}{*}{ FFM (kg) } & & 60.4 & 58.9 & 57.7 & 59.5 & & & \\
\hline & Difference & & -1.5 & -2.7 & -0.9 & & & \\
\hline & $\%$ difference & & $-2.5 \%$ & $-4.5 \%$ & $-1.5 \%$ & & & \\
\hline \multirow[t]{3}{*}{ Albumin ( $g /$ ) } & & 44 & 38 & 41 & 43 & & & \\
\hline & Difference & & -6 & -3 & -1 & & & \\
\hline & $\%$ difference & & $-13.6 \%$ & $-6.8 \%$ & $-2.3 \%$ & & & \\
\hline \multirow[t]{3}{*}{$\mathrm{CRP}(\mathrm{mg} /)$} & & 37 & 211 & 42 & 62 & & & \\
\hline & Difference & & +174 & +5 & +25 & & & \\
\hline & $\%$ difference & & $+470.3 \%$ & $+13.5 \%$ & $+67.6 \%$ & & & \\
\hline Arm B & & Randomisation & Week 20 & $\mathrm{CU} 1$ & CU 2 & CU 3 & CU 4 & CU 5 \\
\hline \multirow[t]{3}{*}{ Weight (kg) } & & 78.6 & 74.6 & & & 77.6 & & \\
\hline & Difference & & -4 & & & -1 & & \\
\hline & $\%$ difference & & $-5.1 \%$ & & & $-1.3 \%$ & & \\
\hline \multirow[t]{3}{*}{ FFM (kg) } & & 47.2 & 46.1 & & & 46.6 & & \\
\hline & Difference & & -1.1 & & & -0.6 & & \\
\hline & $\%$ difference & & $-2.3 \%$ & & & $-1.3 \%$ & & \\
\hline \multirow[t]{3}{*}{ Albumin ( $g /$ ) } & & 40 & 35 & & & 39 & & \\
\hline & Difference & & -5 & & & -1 & & \\
\hline & $\%$ difference & & $-12.5 \%$ & & & $-2.5 \%$ & & \\
\hline \multirow[t]{3}{*}{ CRP (mg/) } & & 5 & 2 & & & 4 & & \\
\hline & Difference & & -3 & & & -1 & & \\
\hline & $\%$ difference & & $-60.0 \%$ & & & $-20.0 \%$ & & \\
\hline
\end{tabular}


Table 5 (continued)

\begin{tabular}{|c|c|c|c|c|c|c|c|c|}
\hline Arm B & & Randomisation & Week 20 & CU 1 & CU 2 & CU 3 & CU 4 & CU 5 \\
\hline \multirow[t]{3}{*}{ Weight (kg) } & & 45.1 & 47.1 & 47.4 & 46.1 & & & \\
\hline & Difference & & +2 & +2.3 & +1 & & & \\
\hline & $\%$ difference & & $+4.4 \%$ & $+5.1 \%$ & $+2.2 \%$ & & & \\
\hline \multirow[t]{3}{*}{ FFM (kg) } & & 39.2 & 39.8 & 39.9 & 37.4 & & & \\
\hline & Difference & & +0.6 & +0.7 & -1.8 & & & \\
\hline & $\%$ difference & & $+1.5 \%$ & $+1.8 \%$ & $-4.6 \%$ & & & \\
\hline \multirow[t]{3}{*}{ Albumin ( $\mathrm{g} /$ ) } & & 25 & 27 & 22 & 23 & & & \\
\hline & Difference & & +2 & -3 & -2 & & & \\
\hline & $\%$ difference & & $+8.0 \%$ & $-12.0 \%$ & $-8.0 \%$ & & & \\
\hline \multirow[t]{3}{*}{ CRP (mg/) } & & 55 & 46 & 37 & 61 & & & \\
\hline & Difference & & -9 & -18 & +6 & & & \\
\hline & $\%$ difference & & $-1.4 \%$ & $-32.7 \%$ & $+10.9 \%$ & & & \\
\hline
\end{tabular}

$\mathrm{FFM}=$ fat free mass, $\mathrm{CRP}=\mathrm{C}-$ reactive protein, $\mathrm{CU}=$ compassionate use

At CU 1 there was a mean percentage albumin change of $-12.9 \%$ (range -20.0 to $-6.8 \%, n=3$ ), with a mean CRP change of $+78.8 \%$ (range -32.7 to $+255.6 \%, n=3$ ). At $\mathrm{CU} 2$ there was a mean percentage albumin change of $-8.2 \%$ (range -14.3 to $-2.3 \%, n=3$ ), with a mean CRP change of $+103.9 \%$ (range +10.9 to $+233.3 \%, n=3)$. At CU 3 there was a mean percentage albumin change of $-2.7 \%$ $(-2.9 \%,-2.5 \%, \mathrm{n}=2)$, with a mean CRP change of $+12.2 \%$ $(-20.0 \%,+44.0 \%, n=2)$. For the remaining participant results at CU 4 there was an albumin change of $0 \%$ and a CRP change of $+255.6 \%$. At CU 5 there was an albumin change of $-5.7 \%$ and a CRP change of $+200.0 \%$. Percentage change was high for one participant, who had a $20.0 \%$ reduction in their albumin level during the first cycle of second-line chemotherapy. It is difficult to discuss trends in CRP levels during compassionate use, due to some participants receiving corticosteroids during chemotherapy and radiotherapy; therefore, this not further discussed here.

High scores on the primary endpoint acceptable questionnaire showed that the participants found engaging in the PRT sessions acceptable. At each session, participants were assessed, and the exercise programme was adapted. Results show that both Arm A participants that transferred onto PRT sessions achieved the planned programme and BORG-Rating of perceived exertion (BORG RPE) 11: 'light' at the end of phase I/week 4 (CU 1=total 24 weeks). The designed low volume, low intensity to a moderate volume, and moderate-high intensity training programmes were both safe and acceptable within a NSCLC end-stage cachectic population. Additionally, it was possible to modify the exercise prescription at different time points for each participant, in an individual adaptive manner, due to ongoing chemotherapy, radiotherapy or further disease progression.

As per Figure 2, there was an overall mean time on the main ACCeRT study + CU of 218 days (range 165 to 305 ) and mean overall survival of 257 days (range 196 to still alive). Interestingly, one participant had relevant stable body weight as well as ECOG-PS and was offered a second line of chemotherapy during $\mathrm{CU}$.

There were no treatment-related deaths, nor exercise-related events, seen within both the main study and during compassionate use. As per the published main study results, there was one possible case of study medication induced atrial fibrillation within one Arm A participant. $^{14}$ 


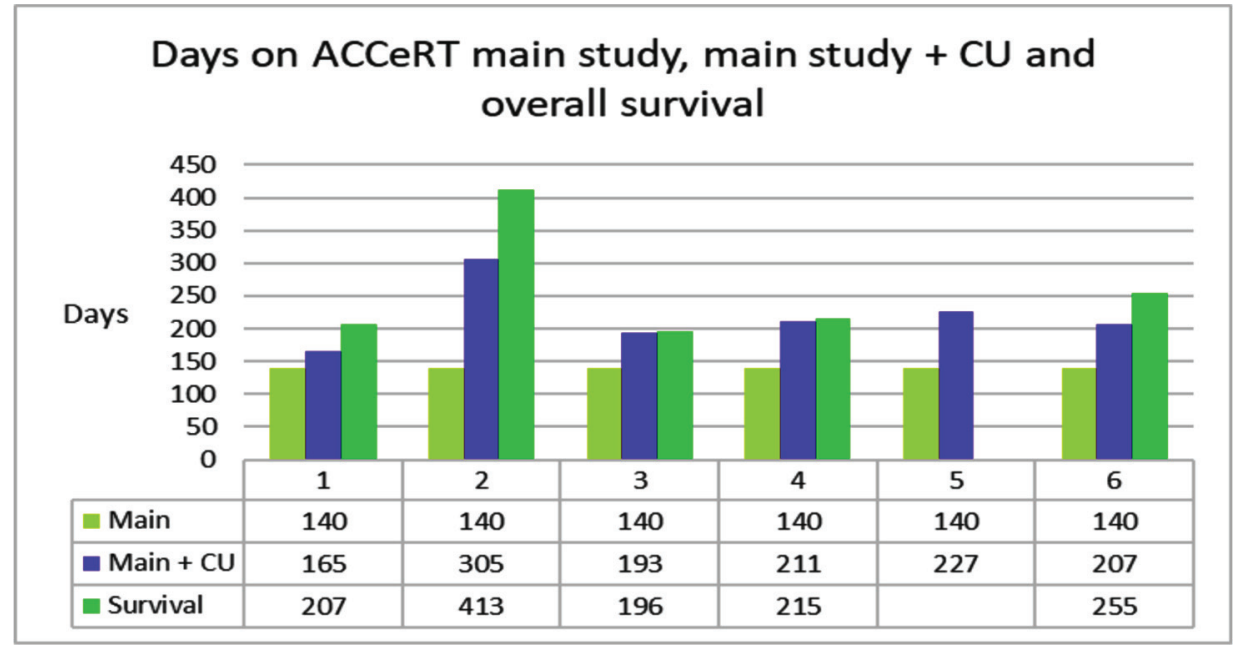

*One participant (participant 5) still alive at time of submission.

ACCeRT=Auckland's Cancer Cachexia evaluating Resistance Training, $\mathrm{CU}=$ compassionate use

Figure 2 Individual participants days on Auckland's Cancer Cachexia evaluating Resistance Training main study plus compassionate use, and overall survival from main study entry

In summary, the first Arm A participant chose to continue with the daily dose of EPA and to begin PRT and EAA only. This participant was continuing on daily diclofenac 100 mg sustained-release for bilateral hip osteoarthritis pre-study entry and completed compassionate use for 25 days and five PRT sessions. Compassionate treatment and PRT was discontinued by the study team at week 23 (week 20 main study + week $3 \mathrm{CU}$ ), due to dislocation of the hip and pelvic pain, and was required to remain in hospital for an extended time. Last recorded ECOG PS=1: there were no other data collected for this participant. The second Arm A participant chose to continue with the daily dose of EPA, celecoxib and to begin PRT only. EAA was declined, due to all the other medications this participant was taking. This participant decided to stop EPA after 8 days of compassionate use, with a compliance of $4.8 \%$. They had completed compassionate use for 165 days and 48 PRT sessions. Compassionate treatment and PRT was discontinued at this participants request at week 44 (week 20 main study + week $24 \mathrm{CU}$ ), due to moving to a property outside of the city. Last recorded ECOG PS=2. The first Arm B participant chose to continue with the daily dose of EPA, celecoxib, PRT and EAA, and completed compassionate use for 53 days and 16 PRT sessions: compassionate treatment and PRT was discontinued at week 28 (20 main study + week $8 \mathrm{CU}$ ) due to death. Last recorded ECOG $\mathrm{PS}=2$ : there were no other data collected for this participant. The second Arm B participant chose to continue with the daily dose of EPA, celecoxib, PRT and EAA, and completed compassionate use for 71 days and 21 PRT sessions. Compassionate treatment and PRT was discontinued at week 31 (week 20 main study + week 11 $\mathrm{CU})$ due to death. Last recorded ECOG $P S=2$. The third Arm B participant stopped EPA at week 11, and received a reduced dose of EAA from week 3 in the main study, and completed compassionate use for 87 days and 25 PRT (32.0\%) sessions. This was due to the logistics of caring for a young family, and attending exercise sessions. Compassionate treatment and PRT was discontinued at the participants request at week 33 (week 20 main study 
+ week $13 \mathrm{CU}$ ) due to moving to another island. Last recorded ECOG PS=2. The fourth Arm B participant chose to continue with the daily dose of EPA, celecoxib, PRT and $E A A$, and completed compassionate use for 67 days and 20 PRT sessions. Compassionate treatment and PRT was discontinued by the study team at week 10 (week 20 main study + week $10 \mathrm{CU}$ ), due to progression of disease and the requirement for hospice respite care. Last recorded $E C O G P S=2$.

\section{Discussion}

The continuation of the ACCeRT study regimen under compassionate use further demonstrates the feasibility and acceptability in patients with refractory cancer cachexia and NSCLC. The multi-targeted regimen has shown further safety without any exercise-induced adverse events.

It was observed that three of the four participants in the $24^{\text {th }}$ week of the intervention still experienced an overall loss of total body weight and FFM. The additional data gained post week 20/EOT visit helps support the longer study time period utilised within the main ACCeRT study, along with gaining new data in this population over an extended time period.

Median survival within the ACCeRT main study of 96 days within Arm A (EPA + celecoxib), versus 136 days within Arm B (EPA, celecoxib, PRT + EAA), versus 257 days in the main ACCeRT study + CU (one participant still alive at submission), is comparable to the followings of two randomized controlled trials (RCT) in late cachexia/ refractory cachexia; wherein anti-cancer treatment was not permitted. Gordon et al., investigated the use of $200 \mathrm{mg}$ thalidomide OD verses a placebo in advanced pancreatic cancer patients, with at least a $10.0 \%$ weight loss over the previous six months; with a planned study period of 24 weeks. Fifty patients were randomised (1:1), with 20 participants achieving eight weeks of treatment. There was a median survival of 148 days within the thalidomide group versus 110 days within the placebo group $(p-v a l u e=0.450) .{ }^{15}$

This was followed by the RCT of EPA versus placebo within lung and gastrointestinal cancer patients, with $\geq 5.0 \%$ of weight loss of pre-illness stable weight. Participants were randomised to either $4 \mathrm{~g}$ EPA, $2 \mathrm{~g}$ EPA, or a placebo OD. There was a study period of eight weeks, with participants permitted to continue with EPA post EOT under compassionate use. ${ }^{16}$ Five hundred and eighteen patients were randomised (1:1:1), with $50.0 \%$ achieving eight weeks of treatment. There was a median overall survival of 142 days within $4 \mathrm{~g}$ EPA, versus 155 days within $2 \mathrm{~g}$ EPA, versus 140 days within the placebo group ( $p$-value=0.750). This is in contrast to the survival data within the ROMANA 1 and 2 studies. ${ }^{17,18}$ Whereas, these results showed no difference between groups with median survival over one year of 8.9 months ( 270 days) within the anamorelin group versus 9.2 months ( 280 days) within the placebo group ( $p$-value=0.470). Additionally, similar survival rates were seen between groups, with deaths of $10.5 \%$ in the anamorelin group versus $13.8 \%$ within the placebo group in the ROMANA 3 extension study. ${ }^{18}$

Other studies, within the pre-cachexia/cachexia phase, have included the Espindolol study, which again showed no significant difference in survival between the high-dose group (61 weeks, 427 days), low-dose group (50.9 weeks, 356 days) and placebo group (42.3 weeks, 296 days)..$^{19}$ The only other multi-targeted study (PreMENAC) showed a median survival of 10 months ( 304 days) within the treatment group versus 8 months ( 243 days) within the control group ( $p$-value $=0.570) .{ }^{11}$ A nonsignificant difference in overall survival was seen between groups in the phase II Enobosarm study, again in patients with pre-cachexia/cachexia. ${ }^{20}$ Interestingly, none of the above studies have shown an overall survival benefit by addressing pre-cachexia/cachexia in patients undergoing anti-cancer treatment. 
ACCeRT is the first study to investigate a multitargeted regimen; including the use of exercise, in a refractory cachexia population. High mean acceptability scores of 4 and 5 were maintained throughout $\mathrm{CU}$.

The ACCeRT study was appropriately designed to evaluate the feasibility of a conservative PRT protocol (given cachexia patient considerations). As PRT was well tolerated during the $\mathrm{CU}$ phase, future studies should look to optimise the PRT to minimise muscle wasting, and maintain patient functional outcomes e.g., activities of daily living, and fatigue.

The ACCeRT CU data has a number of limitations. Firstly, the attrition rate within both Arms within the main study, with two allocated to Arm A and four allocated to Arm B proceeding onto this compassionate use study. Interestingly, participants who continued post week 12 study visit, then went on and completed the week 20/EOT study visit and then further continued with post study CU. Secondly, it must be acknowledged that the open-label design, and that participants could decide on which elements of the regimen to continue with increases the risk of bias of these results. Thirdly, CU body composition was limited to BIA data only, additional CU MRI analysis would have strengthened the skeletal muscle changes.

\section{Conclusion}

In conclusion ACCeRT is the first study to utilise a multi-targeted regimen in the refractory cancer population; therefore, a comparison with other research studies cannot be made at this point. The post main ACCeRT study CU results indicate that patients may benefit from being enrolled onto a multi-targeted cachexia symptom management treatment regimen, even during the late/refractory stage.

\section{Acknowledgement}

We wish to thank all the participants and their whānau (family/community) for participating in the ACCeRT study, along with the support provided by The University of Auckland Centre for Advanced MRI (CAMRI).

\section{Funding sources}

The University of Auckland and the Louisa \& Patrick Emmet Murphy Foundation, Managed by Public Trust (New Zealand) for their generous grants to accomplish this clinical study. Medication supply Pfizer Australia and New Zealand for donated celecoxib (Celebrex), Metagenics (Aust) Pty Ltd for donated EPA, Musashi (Notting Hill, Australia) for the donated essential amino acids. Study completed and registered with Australian and New Zealand Clinical Trials Registry (ANZCTR) number 12611000870954.

\section{Conflict of interest}

All authors declare that they have no competing interests.

\section{References}

1. Vagnildhaug OM, Balstad TR, Almberg SS, Brunelli C, Knudsen AK, Kaasa $S$ et al. A cross-sectional study examining the prevalence of cachexia and areas of unmet need in patients with cancer. Support Care Cancer 2018;26:187180.

2. Dewys WD, Begg C, Lavin PT, Band PR, Bennett JM, Bertino JR, et al. Prognostic effect of weight loss prior to chemotherapy in cancer patients. Am J Med 1980;69:491-7.

3. Andreyev HJN, Norman AR, Oates J, Cunningham D. Why do patients with weight loss have a worse outcome when undergoing chemotherapy for gastrointestinal malignancies? Eur J Cancer 1998;34:503-9.

4. McQuade JL, Daniel CR, Hess KR, Mak C, Wang DY, Rai $\mathrm{RR}$, et al. Association of body-mass index and outcomes in patients with metastatic melanoma treated with targeted therapy, immunotherapy, or chemotherapy: a retrospective, multicohort analysis. Lancet Oncol 2018;19:310-22.

5. Lennon H, Sperrin M, Badrick E, Renehan AG. The obesity paradox in cancer: a review. Curr Oncol Rep 2016;18:1-8.

6. Greenlee H, Unger JM, LeBlanc M, Ramsey S, Hershman DL. Association between body mass index and vancer durvival in 
a pooled analysis of 22 clinical trials. Cancer Epidemio Biomarkers Prev 2017;26:21-9.

7. Coss CC, Clinton SK, Phelps MA. Cachectic Cancer Patients: Immune to Checkpoint Inhibitor Therapy?. Clin Cancer Res 2018;24:5787-9

8. Turner DC, Kondic AG, Anderson KM, Robinson AG, Garon EB, Riess JW, et al. Pembrolizumab exposure-response assessments challenged by association of cancer cachexia and catabolic clearance. Clin Cancer Res 2018;24:5841-9

9. Fearon $\mathrm{KCH}$, Strasser F, Anker SD, Bosaeus I, Bruera E, Fainsinger $\mathrm{RL}$ et al. Definition and classification of cancer cachexia: an international consensus. Lancet Oncol 2011;12: 489-95.

10. Muscaritoli M, Bossola M, Aversa Z, Bellantone R, RossiFanelli F. Prevention and treatment of cancer cachexia: New insights into an old problem. Eur J Cancer 2006;42:31-41.

11. Solheim TS, Laird BJA, Balstad TR, Stene GB, Bye A, Johns $\mathrm{N}$, et al. A randomized phase II feasibility trial of a multimoda intervention for the management of cachexia in lung and pancreatic cancer. J Cachexia Sarcopenia Muscle 2017;8: 778-88.

12. Wakabayashi $H$, Arai $H$, Inui $A$. The regulatory approval of anamorelin for treatment of cachexia in patients with non-small cell lung cancer, gastric cancer, pancreatic cancer, and colorectal cancer in Japan: facts and numbers. J Cachexia Sarcopenia Muscle 2021;12:14-6.

13. Rogers ES, MacLeod RD, Stewart J, Bird SP, Keogh JWL. A randomised feasibility study of EPA and Cox-2 inhibitor (Celebrex) versus EPA, Cox-2 inhibitor (Celebrex), resistance training followed by ingestion of essential amino acids high in leucine in NSCLC cachectic patients - ACCeRT study. BMC Cancer 2011;11:493.

14. Rogers ES, Sasidharan R, Sequeira GM, Wood MR, Bird SP,
Keogh JWL, et al. A multi-targeted treatment approach to cancer cachexia: Auckland's Cancer Cachexia evaluating Resistance Training (ACCeRT) trial. JCSM Rapid Commun 2020;3:11-24.

15. Gordon JN, Trebble TM, Ellis RD, Duncan HD, Johns T, Goggin PM. Thalidomide in the treatment of cancer cachexia: a randomised placebo controlled trial. Gut 2005;54:540-5.

16. Fearon $\mathrm{KCH}$, Barber MD, Moses AG, Ahmedzai SH, Taylor GS, Tisdale MJ, et al. Double-blind, placebo-controlled, randomized study of eicosapentaenoic acid diester in patients with cancer cachexia. J Clin Oncol 2006;24:3401-7.

17. Temel JS, Abernethy AP, Currow DC, Friend J, Duus EM, Yan $\mathrm{Y}$, et al. Anamorelin in patients with non-small-cell lung cancer and cachexia (ROMANA 1 and ROMANA 2): results from two randomised, double-blind, phase 3 trials. Lancet Oncol 2016;18:519-31.

18. Currow D, Temel JS, Abernethy A, Milanowski J, Friend J, Fearon $\mathrm{KCH}$. ROMANA 3: a phase 3 safety extension study of anamorelin in advanced non-small cell lung cancer (NSCLC) patients with cachexia. Ann Oncol 2017;28:1949-56.

19. Stewart Coats AJ, Ho GF, Prabhash K, von Haehling S, Tilson J, Brown R, et al. Espindolol for the treatment and prevention of cachexia in patients with stage III/V non small cell lung cancer or colorectal cancer: a randomized, double blind, placebo controlled, international multicentre phase II study (the ACT ONE trial). J Cachexia Sarcopenia Muscle 2016;7:355-65.

20. Dobs AS, Boccia RV, Croot CC, Gabrail NY, Dalton JT, Hancock ML et al. Effects of enobosarm on muscle wasting and physical function in patients with cancer: a double-blind, randomised controlled phase 2 trial. Lancet Oncol 2013;14:33545. 